\title{
Management of Concomitant Cancer and Abdominal Aortic Aneurysm
}

\author{
Abdullah Jibawi, Islam Ahmed, Karim El-Sakka, and Syed Waquar Yusuf
}

The Vascular Unit, Brighton and Sussex University Hospital, Brighton BN25BE, UK

Correspondence should be addressed to Abdullah Jibawi, abdjibawi@doctors.org.uk and Syed Waquar Yusuf, swyusuf@hotmail.com

Received 15 October 2010; Revised 4 January 2011; Accepted 23 February 2011

Academic Editor: Syed Wamique Yusuf

Copyright (C) 2011 Abdullah Jibawi et al. This is an open access article distributed under the Creative Commons Attribution License, which permits unrestricted use, distribution, and reproduction in any medium, provided the original work is properly cited.

Background. The coexistence of neoplasm and abdominal aortic aneurysm (AAA) presents a real management challenge. This paper reviews the literature on the prevalence, diagnosis, and management dilemmas of concurrent visceral malignancy and abdominal aortic aneurysm. Method. The MEDLINE and HIGHWIRE databases (1966-present) were searched. Papers detailing relevant data were assessed for quality and validity. All case series, review articles, and references of such articles were searched for additional relevant papers. Results. Current challenges in decision making, the effect of major body-cavity surgery on an untreated aneurysm, the effects of major vascular surgery on the treatment of malignancy, the use of EVAR (endovascular aortic aneurysm repair) as a fairly low-risk procedure and its role in the management of malignancy, and the effect of other challenging issues such as the use of adjuvant therapy, and patients informed decision-making were reviewed and discussed. Conclusion. In synchronous malignancy and abdominal aortic aneurysm, the most life-threatening lesion should be addressed first. Endovascular aneurysm repair where possible, followed by malignancy resection, is becoming the preferred initial treatment choice in most centres.

\section{Background}

Malignancy and aortic aneurysms are common diseases, particularly among aging population. Nearly three quarters of malignant cases are diagnosed in people aged 60 years and over [1]. Abdominal aortic aneurysms occur in about $7-8 \%$ of male population over the age of 65 years and is rare under the age of 55 years [2]. The coexistence of malignant disease and aortic aneurysm presents a real management challenge, especially in establishing the therapeutic priorities and the ideal treatment approach. This study aims at reviewing the diagnosis modalities and treatment options and the changes that have taken place with the advances in the management of both cancer and aortic aneurysms, particularly with the development of minimally invasive procedures.

\section{Design and Methods}

The MEDLINE and HIGHWIRE databases were searched using the terms ("neoplasms" [MeSH Terms] or "neo-
plasms"[All Fields] or "cancer"[All Fields]) and ("aortic aneurysm"[MeSH Terms] or "aortic"[All Fields]) and ("aneurysm”[All Fields] or "aortic aneurysm”[All Fields]). All abstracts from the English language articles and foreign language articles were examined by a single reviewer. Papers detailing relevant data were assessed for quality and relevance independently by two separate reviewers. All case series, review articles, and references of such articles were searched for additional relevant papers. Papers that outlined their relevant experience and outcome of their approach sufficiently to allow comparison were included; articles that failed to detail the effect of treatment approach on outcome were excluded. A total of 139 articles were identified. Of these 139 papers, 75 were case series, 57 were case reports, and 5 were discussion papers on various aspects of aortic aneurysm and cancer and all were included in this paper.

\section{Incidence}

Although the true incidence of concomitant malignancy and aortic aneurysm is difficult to establish, most centers 
report a low incidence of intra-abdominal malignancy in patients with abdominal aortic aneurysm. Malignancies were found in $4 \%$ of AAA cases in one of the earlier reviews covering a period of 22 years (Table 1 ). Some authors report a much higher incidence rate, with up to $14 \%$ of AAA cases associated with malignancy in one series published in Japan (Table 1). Based on the natural history of AAA simulated to match the age-specific prevalence rate for male subjects, AAA was estimated to coincidentally occur in $8.3 \%$ of patients with intra-abdominal malignancy. This includes colorectal cancers detected using virtual colonoscopy [3].

\section{Aetiology}

Aortic aneurysms are due to degenerative changes in the aortic wall. Some reports have found an association between the presence of cancer and mycotic aortic aneurysm. For instance, Mycobacterium bovis was found in the wall of a ruptured abdominal aortic and femoral artery aneurysm following intravesical bacillus Calmette-Guérin (BCG) therapy for bladder cancer $[14,15]$. Listeria monocytogenes infection was also found in the wall of a resected thoracic aneurysm two months before advanced rectal cancer was diagnosed. The authors believed that the rectal cancer predisposed the patient to the development of an arterial infection associated with L. monocytogenes [16]. Clostridium septicum mycotic aortic aneurysm has been reported simultaneously with colon cancer [17]. The presence of infection influences the natural history and management. These findings do not establish a causal association but do raise the possibility of an infective aetiology in a very small number of aneurysms.

\section{Clinical Presentation}

Most synchronous abdominal aortic aneurysms and cancers are found incidentally during the investigation or treatment stages. Nevertheless, suspicion of the presence of cancer has been occasionally raised during the workup for aortic aneurysm and vice verse. Table 2 details some examples of such cases.

\section{Investigations}

Computerised tomography (CT) scan is the most common investigation reported to detect the presence of abdominal aortic aneurysms concomitantly with visceral malignancies and vice verse. Occasionally, malignancies are detected incidentally during laparotomy [22] or following an endoscopic procedure such as bronchoscopy [20], upper gastrointestinal [21], or lower gastrointestinal [23] endoscopy. Virtual colonography $[3,24,25]$ or whole-body CT scanning [26] was recommended by some as a more cost-effective strategy to use in population-based screening programmes but not widely accepted.

\section{Management}

There is no consensus on the best management approach for patients with simultaneous aortic aneurysm and malignancy.
Several strategies have been considered, namely, to repair the aneurysm first and treat the malignancy later, to resect the malignancy first and repair the aneurysm later, to undertake both procedures simultaneously, and in some cases to treat the malignancy and manage the aneurysm conservatively.

Aortic aneurysm repair is a prophylactic procedure and is worthwhile where the lifetime risk of rupture exceeds the risk from treatment. The prognosis of cancer is therefore central to the decision making process.

The perceived increase risk of aortic aneurysm rupture following cancer surgery, the significant delay in the treatment of cancer if aneurysm is treated first, and the risk of graft infection are the other important considerations in the management of concomitant aortic aneurysm and cancer.

Table 3 summarizes the different approaches used in the treatment of different patient groups in case series and their outcome.

\section{Discussion}

The treatment of AAA with coexisting malignancy represents a challenging issue to the cancer and vascular specialists in terms of priority, timing, and expected outcome. Most published papers consist of fairly small case series. Prospective randomized controlled trials with adequate statistical power have understandably not been done in this area. In the absence of such level of evidence, one has to combine the best available evidence with a sound clinical judgment applied on each individual case within a multidisciplinary setting.

The management challenge was reflected on a survey of 46 general and vascular professors in the USA in 1985 who gave their responses as to which condition should receive priority of treatment [39]. Excision of the carcinoma first was favored by about a third of them, repair of the aneurysm first was favored by another third, and the remaining third stated that they would withhold a decision until laparotomy was performed. The survey was undertaken in the mid-eighties and a similar survey in the current era would probably show different opinions, especially with the introduction of endovascular and laparoscopic approaches and the availability of advanced preoperative staging techniques and adjuvant therapy.

There is some evidence that abdominal surgery increases the risk of aneurysm rupture, especially when the AAA diameter is over $5 \mathrm{~cm}$. Baxter et al. [11] from the Mayo Clinic reported two AAA ruptures in the immediate postoperative period following 20 CRC (colorectal cancer) excisions (10\% incidence rate). A similar phenomenon was noted by Lin et al. from the Michael E. DeBakey Departement of Surgery, with AAA rupture incidence rate of $6 \%$ following CRC resection [33]. Swanson et al. [40] noted this complication in ten previously asymptomatic patients with aneurysms within 36 days of a prior laparotomy, with a mean AAA diameter of $9.1 \mathrm{~cm}$. On the other hand, Durham et al. [41] prospectively studied 33 patients ( 29 men, 4 women) with a known abdominal aortic aneurysm who underwent 45 operations. The estimated risk of rupture was 3\% of all patients undergoing major operation in this study. It was hypothesized that a reduction in the collagen contents of 
TABLE 1: Prevalence of aortic aneurysm and concomitant malignancy.

\begin{tabular}{|c|c|c|c|c|}
\hline Source & AAA & Malignant disease & Colorectal cancer & $\begin{array}{c}\text { Observation period, } \\
\mathrm{yr}\end{array}$ \\
\hline Szilagyi et al.,1967 [4]* & 803 & $31(3.9)$ & $9(1.2)$ & 22 \\
\hline Nora et al., 1989 [5] & 3500 & $\begin{array}{l}\text { NA (the emphasis is } \\
\text { on CRC) }\end{array}$ & $\begin{array}{l}17(0.5) \text { (those underwent } \\
\text { operations for Ca and AAA) }\end{array}$ & 12 \\
\hline $\begin{array}{l}\text { Morris and Colquitt, } \\
1988[6]\end{array}$ & $\begin{array}{c}158 \text { (looking at all but } \\
\text { with histologically } \\
\text { proven ca) }\end{array}$ & $20(12.7)$ & $6(3.8)$ & 12 \\
\hline Tennant 1990 [7] & 247 & $4(1.6)$ & 0 & 5 \\
\hline Oshodi et al., 2000 [8] & 676 & $8(1.2)$ & $4(0.6)$ & 20 \\
\hline Tsuji et al., 1999 [9] & 162 & $4(2.5)$ & $1(0.6)$ & 10 \\
\hline $\begin{array}{l}\text { Matsumoto et al., } \\
2002[10]\end{array}$ & 260 & $29(11.2)$ & $16(6.2)$ & 14 \\
\hline Baxter et al., 2002 [11] & 10872 & NA & $83(0.8)$ & 15 \\
\hline Yamamoto et al. [12] & $\begin{array}{l}408 \text { (using FOB to } \\
\text { detect CRC) }\end{array}$ & - & $\begin{array}{c}6(1.5 \%) \text { with cancer and } 16 \\
\text { (3.9) with polyps }\end{array}$ & - \\
\hline Onohara et al. [13] & 112 & $16(14 \%)$ & - & - \\
\hline
\end{tabular}

* This study was performed in the pre CT/Duplex era and therefore unlikely to detect cancer. AAA: abdominal aortic aneurysm. CRC: colorectal cancer. NA: not applicable. FOB: faecal occult blood.

TABLE 2: Examples of clinical presentation of aortic aneurysm and/or associated malignancy.

\begin{tabular}{|c|c|c|c|}
\hline Source & Setting & $\begin{array}{l}\text { No. of } \\
\text { cases }\end{array}$ & Mode of presentation* \\
\hline Upchurch and Clair [18] & $\begin{array}{l}\text { Cancer in aortic } \\
\text { aneurysm case }\end{array}$ & 1 case & $\begin{array}{l}\text { Ruptured AAA associated with aortocaval fistula } \\
\text { was complicated by C. Septicum sepsis. CRC was } \\
\text { suspected and found on investigations. }\end{array}$ \\
\hline Tsui, et al. [19] & $\begin{array}{l}\text { Cancer in aortic } \\
\text { aneurysm case }\end{array}$ & 1 case & $\begin{array}{l}\text { Haemoptysis in TAA was initially related to the } \\
\text { aneurysm. Lung cancer was suspected thereafter } \\
\text { and found. }\end{array}$ \\
\hline Van Doorn et al. [20] & $\begin{array}{l}\text { Aortic aneurysm in } \\
\text { cancer case }\end{array}$ & 1 case & $\begin{array}{l}\text { Sepsis and widened mediastinum developed after } \\
\text { CRC operation. Thoracic aneurysm was } \\
\text { suspected and found. }\end{array}$ \\
\hline Mohamed et al. [17] & $\begin{array}{l}\text { Aortic aneurysm in } \\
\text { cancer case }\end{array}$ & 1 case & $\begin{array}{l}\text { CT scan in a patient with sepsis and abdominal } \\
\text { pain revealed ruptured aorta with } \\
\text { pseudoaneurysm. Repeated CT scan in another } \\
\text { centre found CRC in pelvis. }\end{array}$ \\
\hline Sebastian et al. [21] & $\begin{array}{l}\text { Cancer in Aortic } \\
\text { aneurysm case }\end{array}$ & 1 case & $\begin{array}{l}\text { Unsettled dysphagia in TAA was investigated } \\
\text { further. Oesophageal cancer was found. }\end{array}$ \\
\hline
\end{tabular}

*AAA: abdominal aortic aneurysm. CRC: colorectal cancer.

aortic wall following trauma may predispose the aortic aneurysm to rupture, through a mechanism mediated by collagenase and protease activation [40, 42].

The effect of delayed cancer treatment on the longterm outcome remains unclear. Ideally, any delay in cancer management should be avoided. A few months delay before initiating treatment of oesophageal cancer, for example, would have an impact on the stage of the cancer, and thereby on the patients' prognosis [43]. Nevertheless, disease stage at the time of diagnosis is probably the most important influence on survival, and some delay in treatment may not strongly be associated with variation in survival [44]. Delays occurring due to poor organization or the magnitude of the first operation requiring prolonged recovery before the second operation took place, need to be tackled as modern practice allows.
Authors from many large centers (the Mayo Clinic, USA [23], The Cleveland Clinic Foundation, USA [30], the University of Naples Federico II and University of Turin, Italy [36, 37], the Michael E. DeBakey Department of Surgery, USA [8], the Keio University School of Medicine, Japan [13], the Imperial College London, UK [46], the Concord Repatriation General Hospital, Australia [31], and others $[16,45])$ agree that the most life-threatening (or symptomatic) lesion should be addressed first. Large abdominal aortic aneurysms, obstructing colonic cancers, or bleeding gastric cancers, for example, should be treated first where possible. The treatment modality may be a minimally invasive procedure (e.g. stenting for obstructing CRC) that can allow optimization and a better planned therapy [36].

A one-stage operation to repair the aortic aneurysm and resect the malignancy has the advantage of avoiding a 


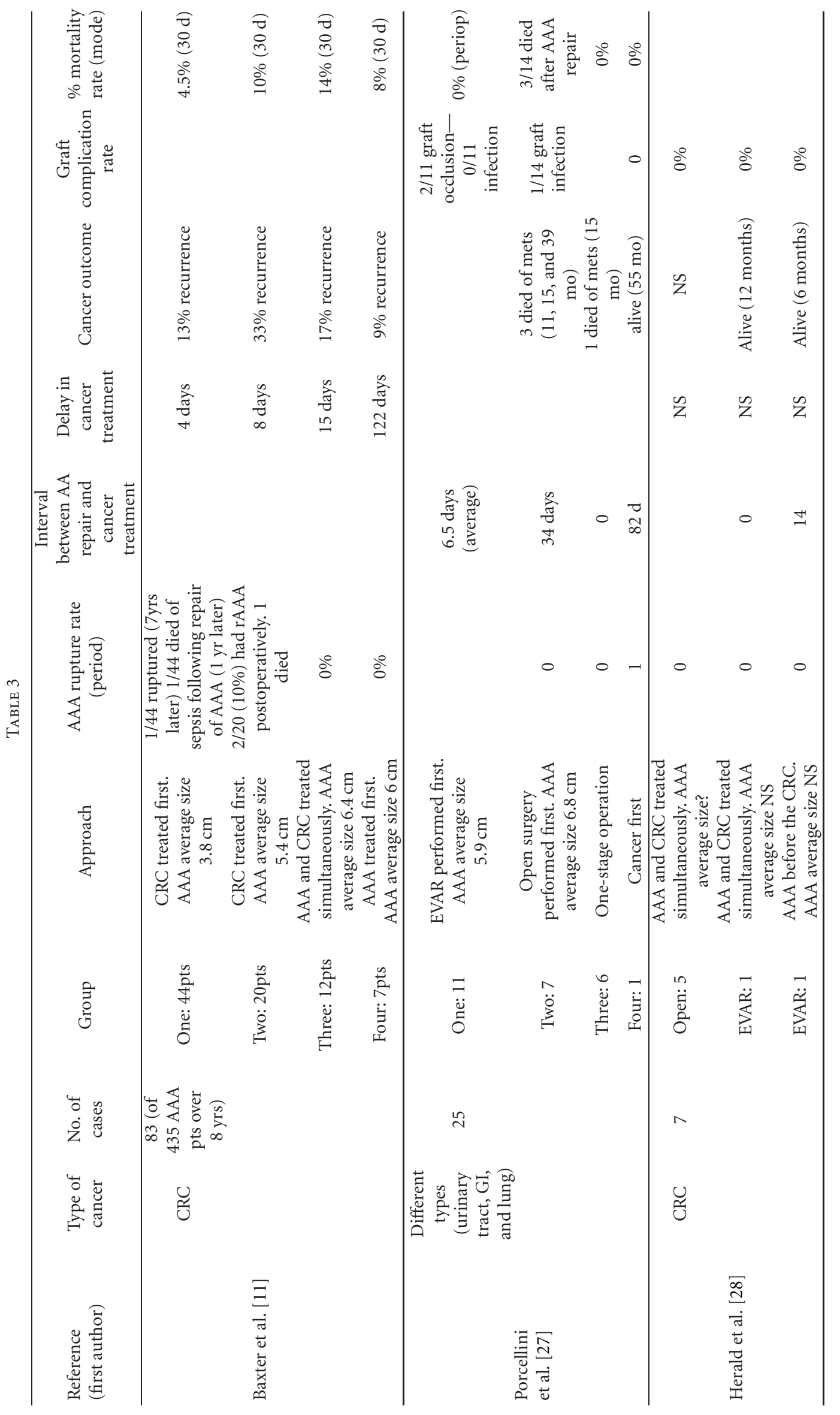




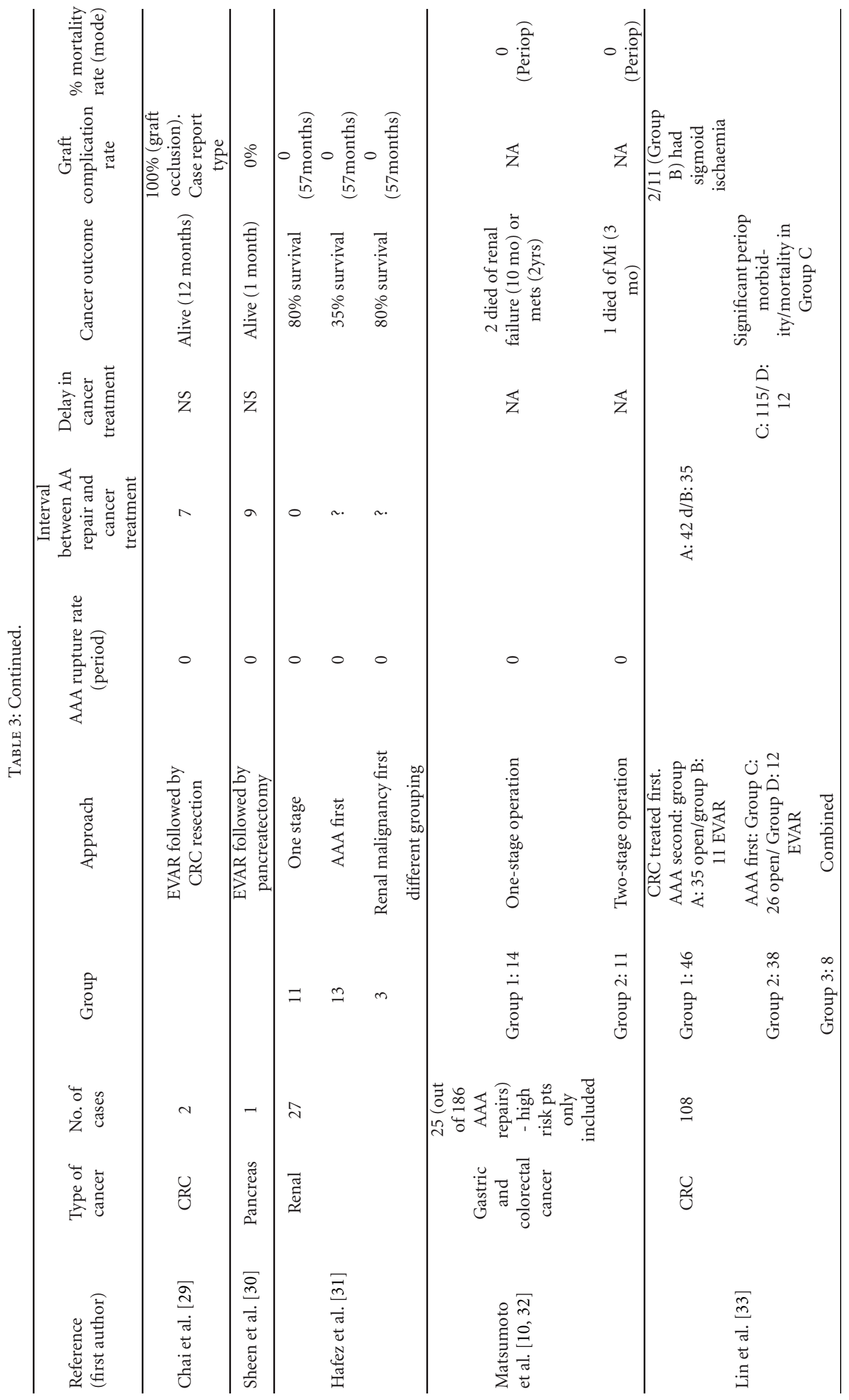




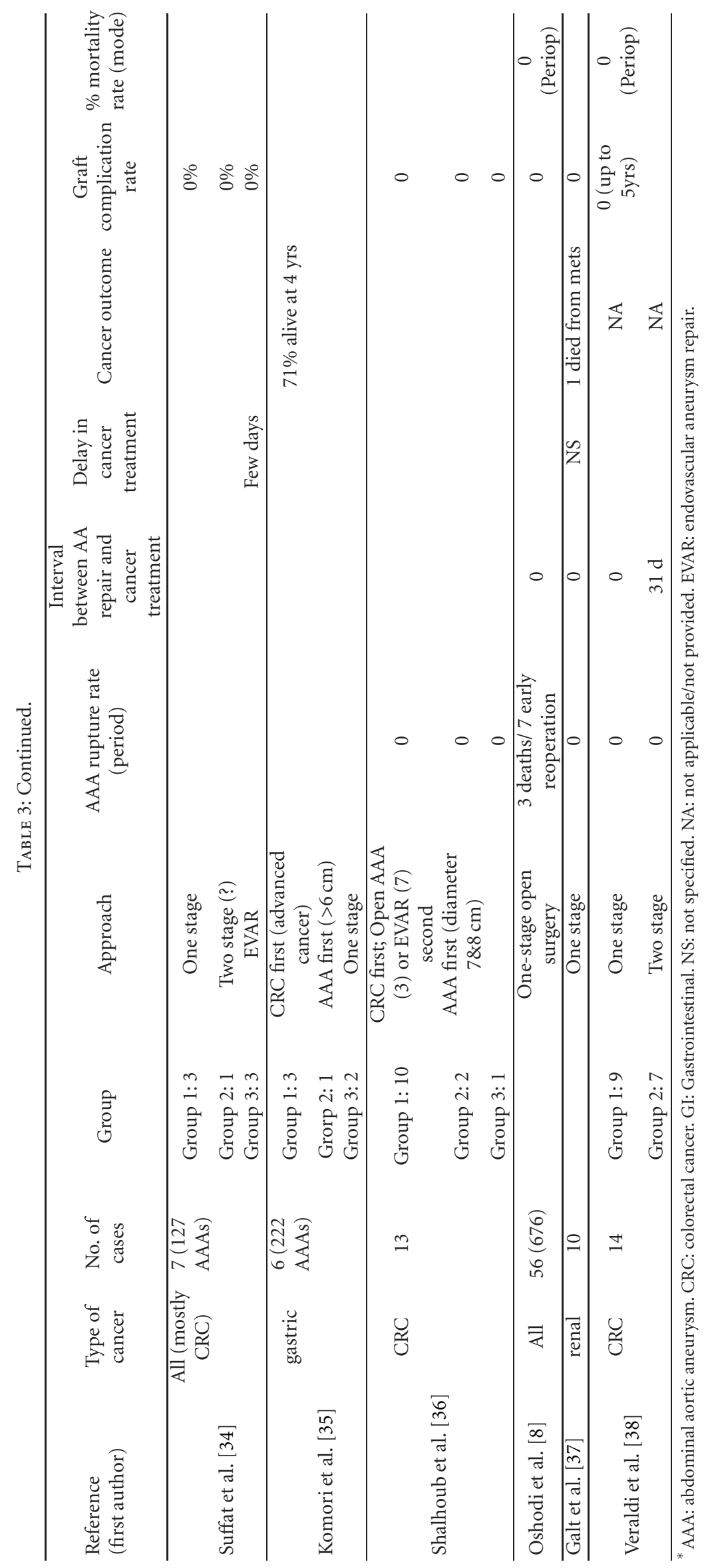


second major abdominal operation, avoiding the potential risk of ruptured aneurysm following the cancer resection stage, avoiding the potential delay in cancer treatment if the aortic aneurysm was addressed first, and avoiding the potential difficulty in dealing with adhesions resulting from an earlier laparotomy [31-33]. Most studies have not shown any significant increase in the risk of graft infection following a one-staged operation (see Table 3, $[8,13,27$, $28,30,31,33-37,45])$. Nevertheless, few reports have documented a possible correlation between graft infection and simultaneous open abdominal surgery [27, 46, 47]. The cumulative morbidity and mortality were significantly higher in one-staged operations when compared to twostaged operations in some reports (Lin et al. [33]), and the one-staged option as a primary approach was unfavorable by some authors accordingly (Lin et al. [33] and Hafez et al. [31]). Combined operation in cases where both lesions pose a life-threatening condition (e.g. large aneurysm with advanced obstructing malignancy) is supported by most authors, providing a very high attention to details in place (thoughtful antibiotic coverage and possible irrigation of operative field with antibiotics [32], usage of antibioticbound grafts [23], good-risk patient selection, verified good colonic blood supply to avoid necrosis, possible use of omental wrap around the vascular graft $[11,32,45]$, possible spray of fibrin glue around anastomosis [32], using extraanatomic bypass instead of a grafted aorta [32], etc.) (Baxter et al. [11], Lin et al. [33], Robinson et al. [23], Suffat et al. [34], and Shalhoub et al. [36]). Some authors, however, remain in favor of a combined operation as a primary management approach to synchronous lesions $[8,13,31$, $32,34]$, especially for fairly clean operations such as gastric $[13,34]$ or urological cancers $[31,37]$.

Staged operation (repair of aortic aneurysm first or resection of malignancy first) has the theoretical advantage of avoiding major longer operation and avoiding the risk of cross-contamination and consequent devastating graft infection. Nevertheless, many reports have shown a significant time delay before the second operation (especially when malignancy is treated first) $[8,11,33,35,38,48]$, a high incidence of catastrophic sequelae (ruptured aortic aneurysm in the case of treating the malignancy first) $[11,23,27,33]$, or a significant decrease in the long-term survival, especially when one pathology was treated eventually [31, 38, 45]. Despite these unfavourable 'side effects' of staged operations, this option (malignancy-first or aorta-first approach) remains the preference for some authors $[6,35,36]$. Few questions such as the impact of aneurysm repair in staged procedure on the timing of adjuvant therapy and the effect of radiotherapy on the EVAR stent need further well-controlled studies in the future.

Endovascular aortic repair (EVAR) in the treatment of concomitant malignancy has attracted much attention more recently [27, 31, 33, 34, 36, 38]. As EVAR does not involve a laparotomy, patients recover quickly and in most cases would be able to undergo the cancer surgery within a couple of weeks if required. Drury and colleagues [49] performed a systematic review and meta-analysis of randomized controlled trials on EVAR and showed a persistent reduction in 30-day mortality (1.6 versus $4.7 \%$ ) and lower incidence of major complications (including cardiac, respiratory, and renal) after EVAR procedures when compared to open procedures [49]. In line with these results, most authors dealing with synchronous AAA and malignancy have found a persistent decrease of the interval period between repairing the aortic aneurysm using EVAR and performing the malignancy operation in the two-staged approach [27, $33,36]$, a persistent reduction in operative morbidity and mortality $[27,33]$ that was sustained up to 3-4 years of followup [27, 33], and a significant reduction in length of hospital stay (unless endoleak has to be ruled out [36, $38])$, and in the intraoperative blood loss [27, 33, 36, 50]. Colonic ischaemia is rare after an EVAR procedure. However, recent prior colonic surgery may predispose patients to such a complication as reported in 2 patients who had right hemicolectomy (with confirmed patent IMA) followed by a staged EVAR (18\% incidence rate) in one report [33]. Lin and colleagues recommended using additional preoperative imaging to confirm the presence of SMA/IMA collateral flow before embarking on such staged operations [33, 38]. Endovascular aneurysm repair, where possible, followed by malignancy operation has become the initial preferred treatment choice in some centers $[31,33,34,38]$. The risk of vascular graft infection is also likely to be less with an EVAR procedure, as there is no risk of direct contamination during the resection of abdominal malignancy.

Patients with advanced/end-stage malignancy and large aortic aneurysm require sound ethical consideration and balanced clinical judgment $[6,8,35,45]$. A good understanding of the expected outcome in each specific cancer type, as well as the presence of other comorbidities (age, physiologic well-being, etc.) is essential for a proper decision making within a multidisciplinary team approach. Aneurysm repair would be considered inappropriate if curative resection is not feasible, even if the EVAR procedure can be carried out with minimum morbidity. The EVAR II trial is particularly helpful in this context. Patients who were deemed unfit for open aortic aneurysm repair were randomly allocated to best medical therapy alone or EVAR and best medical therapy. The trial was weakened by a significant number of patients that crossed over to undergo aneurysm repair. Nevertheless, the trial showed that patients with significant comorbidity do not show any survival benefits with AAA repair [51]. These data can be relevant to cancer patients with poor prognosis who are, on a similar basis, unlikely to benefit from AAA repair.

The need for adjuvant therapy for cancer treatment is another factor to consider when planning for a staged or simultaneous operation [36]. Lauro et al. have raised concerns regarding chemotherapy treatment (associated with aggressive hydration and corticosteroids) in patients known to have AAA $>6 \mathrm{~cm}$. Such treatment may result in enlargement of major arteries and may increase the risk of rupture [42]. There are no documented reports of chemotherapyrelated aneurysm rupture so far. Chemotherapy administered to patients before or after EVAR had no consequences in some reports [36]. Data on the risk of aneurysm rupture due to chemotherapy is limited and should not be an 
important consideration in management decision. It would be entirely reasonable to await the response of chemotherapy and get a better estimate of prognosis before undertaking the repair of aneurysm.

The extent of nodal dissection has significant effect on the overall cancer prognosis, including the need for adjuvant therapy. No technical difficulties or specific limitation have been reported in this respect, including performing a D2 lymphadenectomy in gastric cancer [13] or total mesorectal excision and full node dissection in rectal cancer [38]. However, a one-staged operation appears to be correlated with more D2 dissection and less total gastrectomy rate when compared to staged operations in some series [45]. The effect of paraaortic tissue dissection during open aortic aneurysm repair on the cancer staging or outcome (in terms of cancer dissemination or effect on chemoradiotherapy) or the effect of finding metastatic malignancy following the first stage of treatment (malignancy-first approach) on the cost-effectiveness of aortic-repair stage [36] remains to be investigated further.

Treatment priorities and patients' best interest should be considered when one of the two lesions is found incidentally intraoperatively [38]. Treating the most immediate lifethreatening condition and a two-staged operation might be a sensible option in such circumstances. It should be possible to diagnose aneurysms on CT in most patients preoperatively but malignancy may be occasionally overlooked on the preoperative CT scan for aneurysms. The increasing use of EVAR techniques means that most of these lesions will be detected on preoperative or postoperative surveillance scans and can be treated in the stages.

The management decision for concomitant malignancy and aortic aneurysm needs to be made for each individual case based upon the best estimates of risks of aneurysm rupture, operative risks, prognosis of malignancy, and patient preference. There are multiple variables and it will be difficult to obtain high-level evidence for these patients. It is therefore essential to discuss such cases in a multidisciplinary setting to achieve a consensus opinion before gaining approval from the patient on one option or another.

\section{References}

[1] Cancer Research UK, 2010.

[2] P. E. Norman, K. Jamrozik, M. M. Lawrence-Brown et al., "Population based randomised controlled trial on impact of screening on mortality from abdominal aortic aneurysm," British Medical Journal, vol. 329, no. 7477, pp. 1259-1262, 2004.

[3] C. Hassan, P. Pickhardt, A. Laghi et al., "Computed tomographic colonography to screen for colorectal cancer, extracolonic cancer, and aortic aneurysm: model simulation with cost-effectiveness analysis," Archives of Internal Medicine, vol. 168, no. 7, pp. 696-705, 2008.

[4] D. E. Szilagyi, J. P. Elliott, and R. Berguer, "Coincidental malignancy and abdominal aortic aneurysm. Problems of management," Archives of Surgery, vol. 95, no. 3, pp. 402-412, 1967.

[5] J. D. Nora, P. C. Pairolero, S. Nivatvongs, K. J. Cherry, J. W. Hallett, and P. Gloviczki, "Concomitant abdominal aortic aneurysm and colorectal carcinoma: priority of resection," Journal of Vascular Surgery, vol. 9, no. 5, pp. 630-636, 1989.

[6] D. M. Morris and J. Colquitt, "Concomitant abdominal aortic aneurysm and malignant disease: a difficult management problem," Journal of Surgical Oncology, vol. 39, no. 2, pp. 122$125,1988$.

[7] W. G. B. R. Tennant, "Second intra-abdominal pathology: concomitant or sequential surgery," in The Cause and Management of Aneurysms, R. M. M. J. Greenhalgh, Ed., pp. 321-326, WB Saunders, Philadelphia, Pa, USA, 1990.

[8] T. O. Oshodi, J. S. Abraham, J. K. Brigg, and J. F. Kelly, "Management of co-existing intra-abdominal disease in aortic surgery," European Journal of Vascular and Endovascular Surgery, vol. 19, no. 1, pp. 43-46, 2000.

[9] Y. Tsuji, Y. Watanabe, K. Ataka, A. Sasada, and M. Okada, "Intraabdominal nonvascular operations combined with abdominal aortic aneurysm repair," World Journal of Surgery, vol. 23, no. 5, pp. 469-475, 1999.

[10] K. Matsumoto, T. Murayama, K. Nagasaki et al., "Onestage surgical management of concomitant abdominal aortic aneurysm and gastric or colorectal cancer," World Journal of Surgery, vol. 26, no. 4, pp. 434-437, 2002.

[11] N. N. Baxter, A. A. Noel, K. Cherry, and B. G. Wolff, "Management of patients with colorectal cancer and concomitant abdominal aortic aneurysm," Diseases of the Colon and Rectum, vol. 45, no. 2, pp. 165-170, 2002.

[12] K. Yamamoto, T. Miyata, and H. Nagawa, "The high prevalence of colorectal neoplasms in preoperative patients with abdominal aortic aneurysm or peripheral artery disease," European Journal of Vascular and Endovascular Surgery, vol. 33, no. 4, pp. 397-400, 2007.

[13] T. Onohara, H. Orita, T. Toyohara et al., "Long-term results and prognostic factors after repair of abdominal aortic aneurysm with concomitant malignancy," Journal of Cardiovascular Surgery, vol. 37, no. 1, pp. 1-6, 1996.

[14] C. T. Costiniuk, A. A. Sharapov, G. W. Rose et al., "Mycobacterium bovis abdominal aortic and femoral artery aneurysms following intravesical bacillus Calmette-Guérin therapy for bladder cancer," Cardiovascular Pathology, vol. 19, no. 2, pp. e29-e32, 2010.

[15] G. E. J. Harding and D. K. Lawlor, "Ruptured mycotic abdominal aortic aneurysm secondary to Mycobacterium bovis after intravesical treatment with bacillus CalmetteGuérin," Journal of Vascular Surgery, vol. 46, no. 1, pp. 131134, 2007.

[16] N. Kimura, H. Adachi, K. Adachi, M. Hashimoto, A. Yamaguchi, and T. Ino, "Chronic type A aortic dissection associated with Listeria monocytogenes infection," General Thoracic and Cardiovascular Surgery, vol. 56, no. 8, pp. 417-420, 2008.

[17] H. K. Mohamed, B. M. Elliott, T. E. Brothers, and J. G. Robison, "Suprarenal Clostridium septicum aortitis with rupture and simultaneous colon cancer," Annals of Vascular Surgery, vol. 20, no. 6, pp. 825-829, 2006.

[18] G. R. Upchurch Jr. and D. G. Clair, "Clostridium septicum bacteremia associated with aortic graft infection," Journal of Vascular Surgery, vol. 22, no. 4, pp. 493-495, 1995.

[19] P. Tsui, J. H. Lee, G. MacLennan, and M. Capdeville, "Hemoptysis as an unusual presenting symptom of invasion of a descending thoracic aortic aneurysmal dissection by lung cancer," Texas Heart Institute Journal, vol. 29, no. 2, pp. 136139, 2002.

[20] R. C. Van Doorn, J. Reekers, B. A. J. M. de Mol, H. Obertop, and R. Balm, "Aortoesophageal fistula secondary to mycotic thoracic aortic aneurysm: endovascular repair and transhiatal 
esophagectomy," Journal of Endovascular Therapy, vol. 9, no. 2, pp. 212-217, 2002.

[21] J. Sebastian, V. S. Kirankumar, J. M. Pappachan, S. A. Zachariah, T. R. Radha, and P. Sujathan, "Multifactorial dyspahgia complicated by esophago-bronchial fistula," Journal of Cancer Research and Therapeutics, vol. 3, no. 2, pp. 108-110, 2007.

[22] H. Nakano, K. Esato, M. Ohara, and H. Mohri, "Surgical approach to abdominal aortic aneurysm with malignant alimentary tract tumor: report of three cases," Nippon Geka Gakkai zasshi, vol. 87, no. 7, pp. 803-807, 1986.

[23] G. Robinson, W. Hughes, and E. Lippey, "Abdominal aortic aneurysm and associated colorectal carcinoma: a management problem," Australian and New Zealand Journal of Surgery, vol. 64, no. 7, pp. 475-478, 1994.

[24] P. J. Pickhardt, C. Hassan, A. Laghi, and D. H. Kim, "CT colonography to screen for colorectal cancer and aortic aneurysm in the medicare population: cost-effectiveness analysis," American Journal of Roentgenology, vol. 192, no. 5, pp. 1332-1340, 2009.

[25] J. T. Edwards, C. J. Wood, R. M. Mendelson, and G. M. Forbes, "Extracolonic findings at virtual colonoscopy: implications for screening programs," American Journal of Gastroenterology, vol. 96, no. 10, pp. 3009-3012, 2001.

[26] M. T. Beinfeld, E. Wittenberg, and G. S. Gazelle, "Costeffectiveness of whole-body CT screening," Radiology, vol. 234, no. 2, pp. 415-422, 2005.

[27] M. Porcellini, P. Nastro, U. Bracale, S. Brearley, and P. Giordano, "Endovascular versus open surgical repair of abdominal aortic aneurysm with concomitant malignancy," Journal of Vascular Surgery, vol. 46, no. 1, pp. 16-23, 2007.

[28] J. A. Herald, C. J. Young, G. H. White, and M. J. Solomon, "Endosurgical treatment of synchronous rectal cancer and abdominal aortic aneurysm, without laparotomy," Surgery, vol. 124, no. 5, pp. 932-933, 1998.

[29] C. Y. Chai, P. H. Lin, R. L. Bush, and A. B. Lumsden, "Aortic endograft thrombosis after colorectal surgery in lithotomy position," Journal of Vascular Surgery, vol. 39, no. 5, pp. 1112$1114,2004$.

[30] A. J. Sheen, M. Baguneid, S. Ellenbogen, M. G. Walker, and A. K. Siriwardena, "Sequential endovascular repair and pancreaticoduodenectomy for abdominal aortic aneurysm copresenting with periampullary cancer," Annals of Vascular Surgery, vol. 20, no. 1, pp. 114-116, 2006.

[31] K. S. Hafez, H. A. El Fettouh, A. C. Novick, and K. Ouriel, "Management of synchronous renal neoplasm and abdominal aortic aneurysm," Journal of Vascular Surgery, vol. 32, no. 6, pp. 1102-1110, 2000.

[32] K. Matsumoto, M. Nakamaru, H. Obara et al., "Surgical strategy for abdominal aortic aneurysm with concurrent symptomatic malignancy," World Journal of Surgery, vol. 23, no. 3, pp. 248-251, 1999.

[33] P. H. Lin, N. R. Barshes, D. Albo et al., "Concomitant colorectal cancer and abdominal aortic aneurysm: evolution of treatment paradigm in the endovascular era," Journal of the American College of Surgeons, vol. 206, no. 5, pp. 1065-1073, 2008.

[34] L. P. Suffat, P. Guffanti, F. Rebecchi, P. P. Suffat, and F. Morino, "Abdominal aortic aneurysm and concomitant malignancy: what treatment?" Annali Italiani di Chirurgia, vol. 77, no. 4, pp. 345-349, 2006.

[35] K. Komori, K. Okadome, S. Funahashi, H. Itoh, and K. Sugimachi, "Surgical strategy of concomitant abdominal aortic aneurysm and gastric cancer," Journal of Vascular Surgery, vol. 19, no. 4, pp. 573-576, 1994.

[36] J. Shalhoub, P. Naughton, N. Lau et al., "Concurrent colorectal malignancy and abdominal aortic aneurysm: a multicentre experience and review of the literature," European Journal of Vascular and Endovascular Surgery, vol. 37, no. 5, pp. 544-556, 2009.

[37] S. W. Galt, W. J. McCarthy, W. H. Pearce et al., "Simultaneous abdominal aortic aneurysm repair and nephrectomy for neoplasm," American Journal of Surgery, vol. 170, no. 2, pp. 227-230, 1995.

[38] G. F. Veraldi, S. Tasselli, G. De Manzoni, and C. Cordiano, "Surgical treatment of abdominal aortic aneurysm with concomitant renal cell carcinoma: a single-centre experience with review of the literature," Journal of Cardiovascular Surgery, vol. 47, no. 6, pp. 643-649, 2006.

[39] V. J. Lobbato, R. E. Rothenberg, R. D. LaRaja, and J. Georgiou, "Coexistence of abdominal aortic aneurysm and carcinoma of the colon: a dilemma," Journal of Vascular Surgery, vol. 2, no. 5, pp. 724-726, 1985.

[40] R. J. Swanson, F. N. Littooy, T. K. Hunt, and R. J. Stoney, "Laparotomy as a precipitating factor in the rupture of intraabdominal aneurysms," Archives of Surgery, vol. 115, no. 3, pp. 299-304, 1980.

[41] S. J. Durham, D. L. Steed, H. H. Moosa, M. S. Makaroun, and M. W. Webster, "Probability of rupture of an abdominal aortic aneurysm after an unrelated operative procedure: a prospective study," Journal of Vascular Surgery, vol. 13, no. 2, pp. 248-252, 1991.

[42] S. Lauro, G. Lanzetta, E. Bria, L. Trasatti, A. Gelibter, and A. Vecchione, "Antiblastic chemotherapy in the presence of abdominal aorta aneurysm (AAA): guidelines," Anticancer Research, vol. 19, no. 2, pp. 1439-1444, 1999.

[43] J. Wang, F. Liu, H. Gao et al., "The symptom-to-treatment delay and stage at the time of treatment in cancer of esophagus," Japanese Journal of Clinical Oncology, vol. 38, no. 2, pp. 87-91, 2008.

[44] M. C. Gulliford, A. Petruckevitch, and P. G. J. Burney, "Survival with bladder cancer, evaluation of delay in treatment, type of surgeon, and modality of treatment," British Medical Journal, vol. 303, no. 6800, pp. 437-440, 1991.

[45] C. Pedrazzani, G. F. Veraldi, S. Tasselli et al., "Surgical treatment of gastric cancer with coexistent abdominal aortic aneurysm. Personal experience and literature review," HepatoGastroenterology, vol. 53, no. 72, pp. 973-975, 2006.

[46] V. M. Bernhard, "Management of infected vascular prostheses," Surgical Clinics of North America, vol. 55, no. 6, pp. 14111417, 1975.

[47] F. M. Ameli, M. Weiss, J. L. Provan, and K. W. Johnston, "Safety of cholecystectomy with abdominal aortic surgery," Canadian Journal of Surgery, vol. 30, no. 3, pp. 170-173, 1987.

[48] F. Grego, S. Lepidi, P. Bassi et al., "Simultaneous surgical treatment of abdominal aortic aneurysm and carcinoma of the bladder," Journal of Vascular Surgery, vol. 37, no. 3, pp. 607614, 2003.

[49] D. Drury, J. A. Michaels, L. Jones, and L. Ayiku, "Systematic review of recent evidence for the safety and efficacy of elective endovascular repair in the management of infrarenal abdominal aortic aneurysm," British Journal of Surgery, vol. 92, no. 8, pp. 937-946, 2005.

[50] N. Peppelenbosch, J. Buth, P. L. Harris et al., "Diameter of abdominal aortic aneurysm and outcome of endovascular aneurysm repair: does size matter? A report from 
EUROSTAR," Journal of Vascular Surgery, vol. 39, no. 2, pp. 288-297, 2004.

[51] R. M. Greenhalgh, D. J. Allison, P. R. F. Bell et al., "Endovascular repair of aortic aneurysm in patients physically ineligible for open repair," The New England Journal of Medicine, vol. 362 , no. 20, pp. 1872-1880, 2010. 


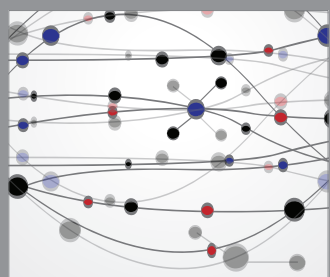

The Scientific World Journal
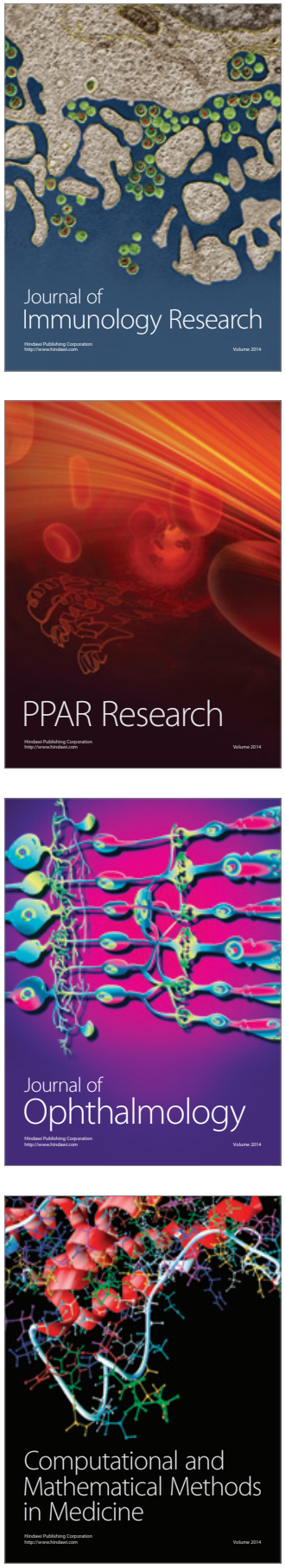

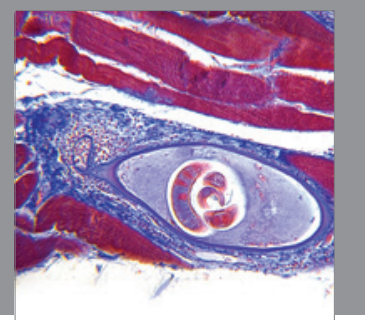

Gastroenterology

Research and Practice
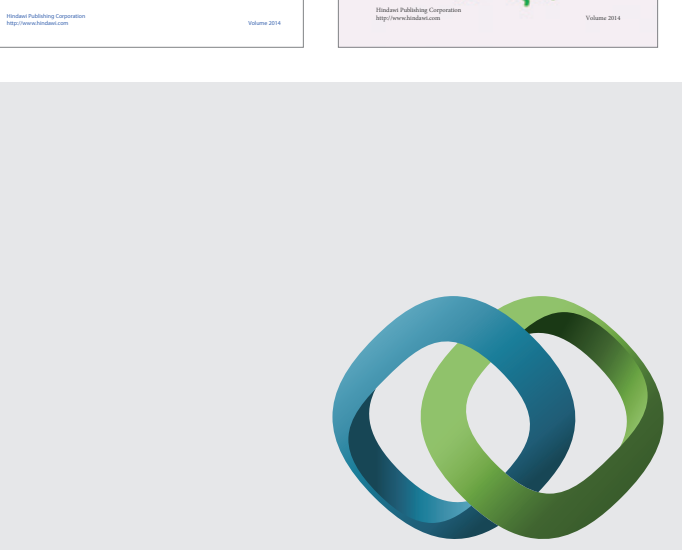

\section{Hindawi}

Submit your manuscripts at

http://www.hindawi.com
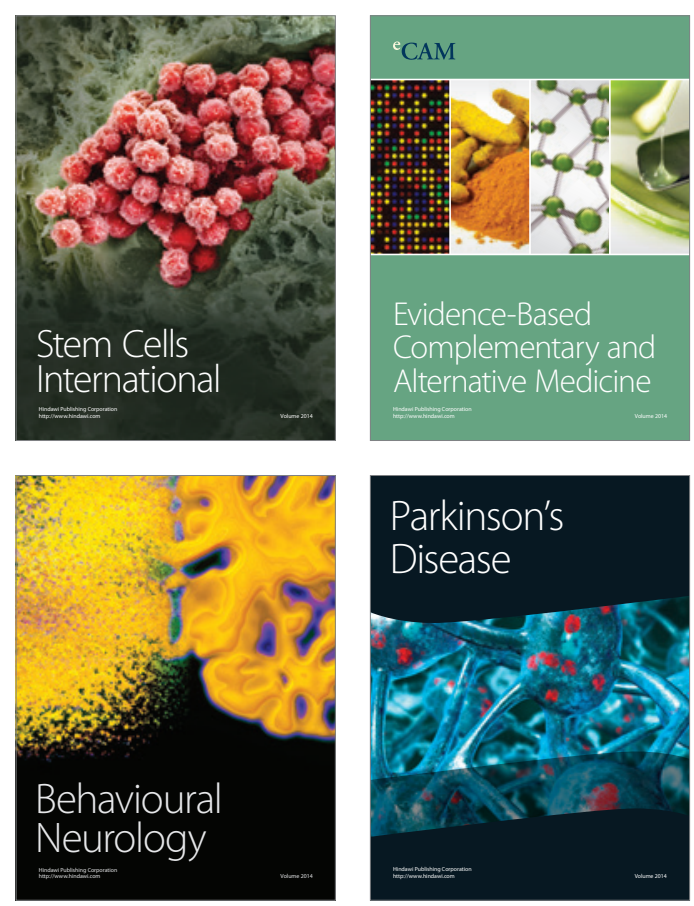

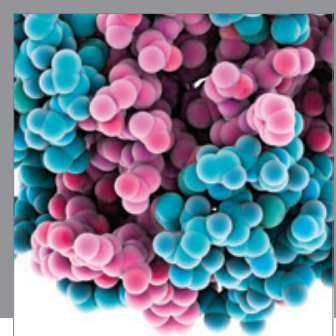

Journal of
Diabetes Research

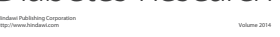

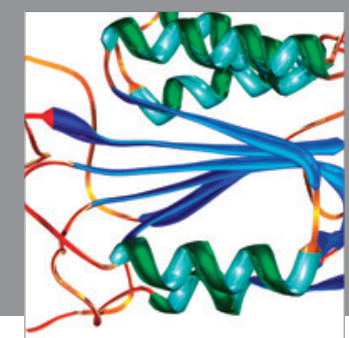

Disease Markers
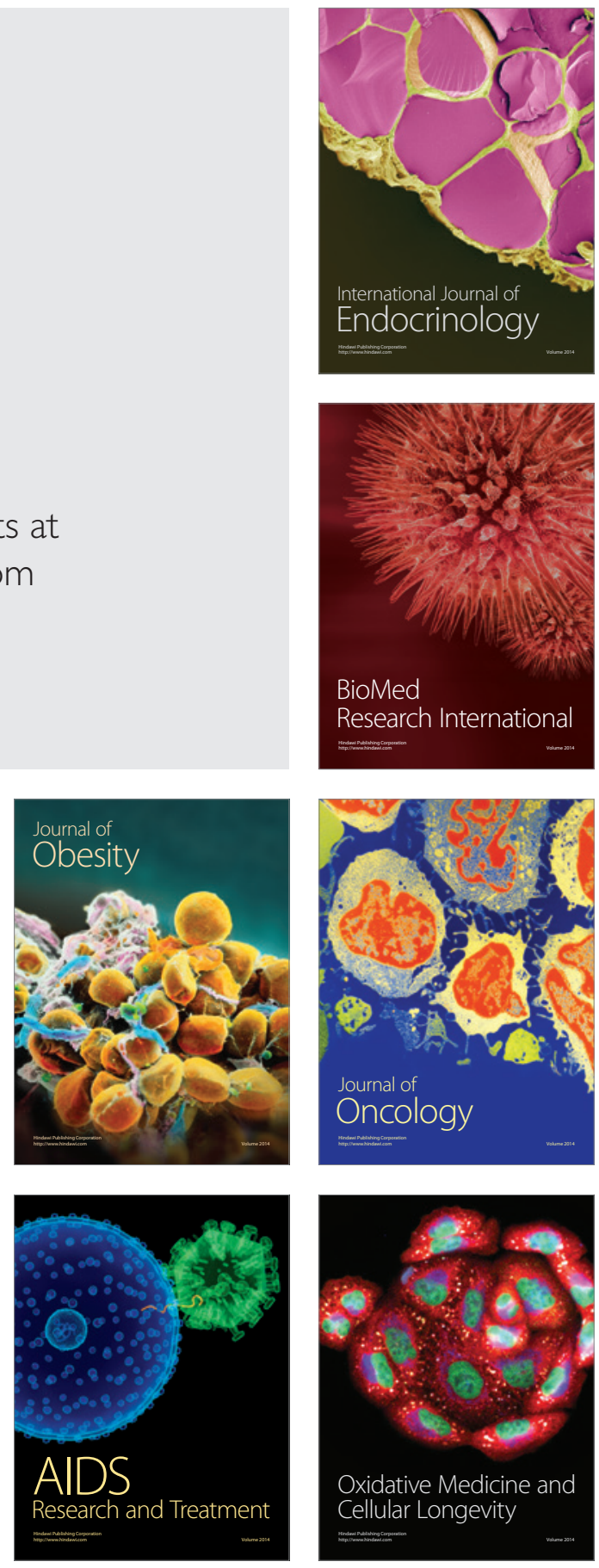